\title{
Degree sum conditions for hamiltonian index
}

\author{
LIU Ze-meng ${ }^{1} \quad$ XIONG Li-ming ${ }^{2}$
}

\begin{abstract}
In this note, we show a sharp lower bound of $\min \left\{\sum_{i=1}^{k} d_{G}\left(u_{i}\right): u_{1} u_{2} \ldots u_{k}\right.$ is a path of (2-)connected $G$ \} on its order such that $(k-1)$-iterated line graphs $L^{k-1}(G)$ are hamiltonian.
\end{abstract}

\section{$\S 1 \quad$ Introduction}

We use Bondy and Murty [2] for terminology and notation not defined here and consider finite simple graphs only. Let $G=(V(G), E(G))$ be a connected graph and $u$ be a vertex of $G$. We use $N_{G}(u)$ to denote the set of vertices which are adjacent with $u$ (also called the neighbors of $u$ ) in the graph $G \cdot d_{G}(u)=\left|N_{G}(u)\right|$ is the degree of $u$ in $G$. Let $S$ be a subset of $V(G)$ (or $E(G))$. The induced subgraph of $G$ is denoted by $G[S]$. We use $K_{n}$ to denote the complete graph of order $n$. The clique $C$ is a subset of $V(G)$ such that $G[C]$ is a complete graph.

The line graph $L(G)$ of $G=(V(G), E(G))$ has $E(G)$ as its vertex set, and two vertices are adjacent in $L(G)$ if and only if the corresponding edges share a common end vertex in $G$. The $m$-iterated line graph $L^{m}(G)$ is defined recursively by $L^{0}(G)=G, L^{1}(G)=L(G)$ and $L^{m}(G)=L\left(L^{m-1}(G)\right)$. The hamiltonian index of a graph $G$, denoted by $h(G)$, is the smallest integer $m$ such that $L^{m}(G)$ is hamiltonian, i.e., it has a spanning cycle.

Chartrand [5] showed that the hamiltonian index for any graph other than a path always exists and that $L(G)$ of a hamiltonian graph $G$ is hamiltonian. For a connected graph that is not a path, Ryjáček, Woeginger and Xiong [8] showed that the problem to decide whether the hamiltonian index of a given graph is less than or equal to a given constant is NP-complete.

Saražin [9] showed that $h(G) \leq n-\Delta(G)$ if $G$ is connected graph of order $n$, later, Xiong [11] improved this result and showed that $h(G) \leq \operatorname{diam}(G)-1$ if $G$ is a connected graph other than a path since $\operatorname{diam}(G)-1 \leq n-\Delta(G)$, where $\operatorname{diam}(G)$ denotes the diameter of a graph $G$. For its other sharp upper bounds and stability, see [4] and [12], and [14], respectively, while its sharp lower bound is also gave in [12]; in [15], you may see its survey paper.

Let $P \subseteq G$ be a path of order $k \geq 1$. By $d_{G}(P)$, we denote the degree of a path $P$. That is, $d_{G}(P)=d_{G}\left(v_{1}\right)+d_{G}\left(v_{1}\right)+\cdots+d_{G}\left(v_{k}\right)$, where $V(P)=\left\{v_{1}, v_{2}, \cdots, v_{k}\right\}$. By $\bar{\sigma}_{k}(G)$,

\footnotetext{
Received: 2019-8-18. Revised: 2020-03-24.

MR Subject Classification: 05C07, 05C45, 05C76.

Keywords: iterated line graph, hamiltonian index, degree sum condition.

Digital Object Identifier(DOI): https://doi.org/10.1007/s11766-021-3885-4.

Supported by the National Natural Science Foundation of China(11871099).

(C)The Author(s) 2021.
} 
we denote $\min \left\{d_{G}(P): P\right.$ is a path of $G$ with $\left.|V(P)|=k\right\}$. Obviously $\delta(G)=\bar{\sigma}_{1}(G)$ and $\delta(L(G))=\bar{\sigma}_{2}(G)-2$ for every nonempty graph $G$.

Dirac [7] showed a very famous result that every graph $G=L^{0}(G)$ of order $n$ with $\delta(G)=$ $\bar{\sigma}_{1}(G) \geq \frac{n}{2}$ is hamiltonian, while Brualdi and Shanny [3] gave a similar result on $L(G)=L^{1}(G)$ involving $\bar{\sigma}_{2}(G)$, which was later improved slightly by Clark [6] for graphs with large order.

Theorem 1 (Brualdi and Shanny, [3]). If $G$ is a graph of order $n \geq 4$ and at least one edge such that $\bar{\sigma}_{2}(G)>n$, then $L(G)$ is hamiltonian.

Theorem 2 (Clark, [6]). If $G$ is a connected graph of order $n \geq 6$ and if

$$
\bar{\sigma}_{2}(G) \geq\left\{\begin{array}{ll}
n-1, & \text { if } n \text { is even } \\
n-2, & \text { if } n \text { is odd }
\end{array},\right.
$$

then $L(G)$ is hamiltonian.

For almost bridgeless graphs (i.e., graphs in which every cut edge is incident with vertex of degree one), Veldman improved the above result to the following theorem which settled a conjecture in [1].

Theorem 3 (Veldman, [10]). Let $G$ be a connected almost bridgeless graph of sufficiently large order $n$ such that $\bar{\sigma}_{2}(G)>2\left(\left\lfloor\frac{n}{5}\right\rfloor-1\right)$, then $L(G)$ is hamiltonian.

In this paper, we consider similar sufficient conditions for $m$-iterated line graphs $L^{m}(G)$ to be hamiltonian for $m \geq 2$ and get the following main results.

Theorem 4. Let $k \geq 3$ be an integer and let $G$ be a connected graph of order $n>k+2$ such that $\bar{\sigma}_{k}(G)>n+k-3$, then $L^{k-1}(G)$ is hamiltonian, i.e., $h(G) \leq k-1$.

Theorem 5. Let $k \geq 3$ be an integer and let $G$ be a 2-connected graph of order $n>6 k+3$ such that $\bar{\sigma}_{k}(G)>\frac{2 n}{5}-\frac{2 k}{5}-\frac{1}{5}$, then $L^{k-1}(G)$ is hamiltonian, i.e., $h(G) \leq k-1$.

\section{$\S 2 \quad$ Preliminaries}

Let $G$ be a graph, define $V_{i}(G)=\left\{v \in V(G): d_{G}(v)=i\right\}$ and $V_{\geq i}(G)=\{v \in V(G)$ : $d_{G}(v) \geq i$. Let $P(u, v)$ denote a path between $u$ and $v$. A branch in $G$ is a nontrivial path with ends not in $V_{2}(G)$ and with internal vertices, if any, that have degree 2 in $G$. By $\mathcal{B}(G)$, we denote the set of branches of $G$. Define $\mathcal{B}_{1}(G)=\left\{B \in \mathcal{B}(G): V(B) \cap V_{1}(G) \neq \emptyset\right\}$. Let $H_{1}$ and $H_{2}$ be two subgraphs of graph $G$. Define $H_{1} \cup H_{2}=G\left[E\left(H_{1}\right) \cup E\left(H_{2}\right)\right], H_{1} \cap H_{2}=G\left[E\left(H_{1}\right) \cap E\left(H_{2}\right)\right]$, $H_{1}-H_{2}=G\left[E\left(H_{1}\right) \backslash E\left(H_{2}\right)\right], H_{1} \triangle H_{2}=G\left[E\left(H_{1}\right) \triangle E\left(H_{2}\right)\right]=G\left[\left(E\left(H_{1}\right) \cup E\left(H_{2}\right)\right) \backslash\left(E\left(H_{1}\right) \cap\right.\right.$ $\left.E\left(H_{2}\right)\right)$ ], respectively. For any $S \subseteq V(G)$, define $H_{1} \cup S$ is a graph with $V\left(H_{1}\right) \cup S$ and $E\left(H_{1}\right)$ as its vertex set and edge set, respectively. The distance $d_{G}\left(H_{1}, H_{2}\right)$ between $H_{1}$ and $H_{2}$ is defined to be $\min \left\{d_{G}\left(v_{1}, v_{2}\right): v_{1} \in V\left(H_{1}\right), v_{2} \in V\left(H_{2}\right)\right\}$, where $d_{G}\left(v_{1}, v_{2}\right)$ denotes the number of edges of a shortest path between $v_{1}$ and $v_{2}$ in $G$.

Xiong and Liu [13] characterized the graphs for which the $s$-iterated line graph is hamiltonian for any integer $s \geq 2$.

Theorem 6 (Xiong and Liu, [13]). Let $G$ be a connected graph that is not a 2-cycle and let $s \geq 2$ be an integer. Then $h(G) \leq s$ if and only if $E U_{s}(G) \neq \emptyset$, where $E U_{s}(G)$ denotes the set of those subgraphs $H$ of a graph $G$ that satisfy the following conditions:

(I) $d_{H}(x) \equiv 0(\bmod 2)$ for every $x \in V(H)$;

(II) $V_{0}(H) \subseteq V_{\geq 3}(G) \subseteq V(H)$; 
(III) $d_{G}\left(H_{1}, H-H_{1}\right) \leq s-1$ for every subgraph $H_{1}$ of $H$;

(IV) $|E(B)| \leq s+1$ for every branch $B \in \mathcal{B}(G)$ with $E(B) \cap E(H)=\emptyset$;

(V) $|E(B)| \leq s$ for every branch $B \in \mathcal{B}_{1}(G)$.

\section{$\S 3 \quad$ Proofs of main results}

Proof of Theorem 4. Choose a subgraph $H$ of $G$ satisfying that:

(1) $d_{H}(x) \equiv 0(\bmod 2)$ for every $x \in V(H)$;

(2) $V_{0}(H) \subseteq V_{\geq 3}(G) \subseteq V(H)$;

(3) subject to (1), (2), $|V(H)|$ is maximized.

By Theorem 6, for $s=k-1$, it suffices to prove that $H \in E U_{k-1}(G)$. By the choice of $H$, $H$ satisfies Conditions (I), (II) of Theorem 6.

We claim that $H$ satisfies Condition (IV) of Theorem 6. That is, $|E(B)| \leq k$ for every $B \in \mathcal{B}(G)$ with $E(B) \cap E(H)=\emptyset$. Suppose otherwise. Then $G$ has a branch $B \in \mathcal{B}(G)$ such that $|E(B)| \geq k+1$ and $E(B) \cap E(H)=\emptyset$. Hence, $B$ contains a path $P$ of order $k$ with $V(P) \subseteq V_{2}(G)$. For $n>k+2, \bar{\sigma}_{k}(G) \leq d_{G}(P)=2 k<n+k-2 \leq \bar{\sigma}_{k}(G)$, a contradiction. We then claim that $H$ satisfies Condition (V) of Theorem 6 . That is, $|E(B)| \leq k-1$ for every $B \in \mathcal{B}_{1}(G)$. Suppose otherwise. Then $G$ has a branch $B \in \mathcal{B}_{1}(G)$ with $|E(B)| \geq k$. Hence, $B$ contains a path $P$ of order $k$ with $\bar{\sigma}_{k}(G) \leq d_{G}(P)=2 k-1<n+k-2 \leq \bar{\sigma}_{k}(G)$, a contradiction.

Then we only need to prove that $H$ satisfies Condition (III) of Theorem 6 , that is, $d_{G}\left(H_{1}, H-\right.$ $\left.H_{1}\right) \leq k-2$ for every subgraph $H_{1}$ of $H$. Suppose otherwise. Then $H$ has a subgraph $H_{1}$ such that $d_{G}\left(H_{1}, H-H_{1}\right) \geq k-1$. Since $G$ is connected and $k \geq 3$, there is at least one path $B_{0}=x_{0} x_{1} \cdots x_{l}$ between $H_{1}$ and $H-H_{1}$ such that $l \geq k-1$ and $x_{0} \in V\left(H_{1}\right)$ and $x_{l} \in V\left(H-H_{1}\right)$. By the choice of $H, B_{0}$ is a branch of $G$. Without loss of generality, we assume that $\left|V\left(H_{1}\right)\right| \leq\left|V\left(H-H_{1}\right)\right|$. Then $\left|V\left(H_{1}\right)\right| \leq\left\lfloor\frac{\mid V(H)\rfloor}{2}\right\rfloor$.

Claim 1. For any vertex $y_{0} \in V(G)-\left(V(H) \bigcup V\left(B_{0}\right)\right)$, if $N_{G}\left(y_{0}\right) \subseteq V(H)$, then $N_{G}\left(y_{0}\right)$ is an independent set.

Proof. Suppose otherwise. Since $y_{0} \notin V(H), d_{G}\left(y_{0}\right) \leq 2$. Since Claim 1 naturally holds when $d_{G}\left(y_{0}\right)=1$, we only need to consider the case when $d_{G}\left(y_{0}\right)=2$. Then $v_{1} v_{2}$ is an edge of $G$ for $N_{G}\left(y_{0}\right)=\left\{v_{1}, v_{2}\right\} \subseteq V(H)$. Then $H^{1}=H \triangle v_{1} v_{2} y_{0} v_{1}$ is a subgraph of $G$ satisfying (1), (2) of (3.1) and $\left|V\left(H^{1}\right)\right|>|V(H)|$, contradicting the choice of $H$ in terms of (3) of (3.1).

Claim 2. $\left|N_{G}\left(x_{0}\right) \bigcap N_{G}\left(x_{l}\right)\right| \leq 1$.

Proof. Suppose otherwise. Then $\left|N_{G}\left(x_{0}\right) \cap N_{G}\left(x_{l}\right)\right| \geq 2$. Then there exists a vertex $x \in N_{G}\left(x_{0}\right) \cap N_{G}\left(x_{l}\right)$ and $x \notin B_{0}$. Then $x_{0} x x_{l}$ is a branch of $G$, denoted by $B_{1}$. Then $C^{2}=B_{0} \bigcup B_{1}$ is a cycle. We have a subgraph $H^{2}=H \triangle C^{2}$ of $G$ satisfying (1), (2) of (3.1) and $\left|V\left(H^{2}\right)\right|>|V(H)|$, contradicting the choice of $H$ in terms of (3) of (3.1).

Claim 3. $V(G)=V(H) \bigcup V\left(B_{0}\right)$.

Proof. Suppose otherwise. Since $d_{G}\left(x_{0}\right) \geq 3, N_{G}\left(x_{0}\right) \neq \emptyset$. We consider two cases.

Case 1. $N_{G}\left(x_{0}\right) \cap V\left(H_{1}\right)=\emptyset$.

Since $d_{G}\left(H_{1}, H-H_{1}\right) \geq k-1 \geq 2, N_{G}\left(x_{0}\right) \subseteq V(G-H)$. Since $d_{G}\left(x_{l}\right) \geq 3,\left|N_{G}\left(x_{l}\right)\right| \geq 3$. By Claim 2, $d_{G}\left(x_{0}\right) \leq n-(k-3+3+1)=n-k-1$. Note that $P=y x_{0} \cdots x_{k-2}$ is a 
path of order $k$, where $y \in N_{G}\left(x_{0}\right)$. Then $d_{G}(y) \leq 2$. However, $\bar{\sigma}_{k}(G) \leq d_{G}\left(P_{0}\right) \leq d_{G}(P) \leq$ $2+n-k-1+2(k-2)=n+k-3<\bar{\sigma}_{k}(G)$, a contradiction.

Case 2. $N_{G}\left(x_{0}\right) \cap V\left(H_{1}\right) \neq \emptyset$.

For $y \in N_{G}\left(x_{0}\right) \cap V\left(H_{1}\right), P=y x_{0} \cdots x_{k-2}$ is a path of order $k$. By Claim $1, N_{G}\left(x_{0}\right) \cap N_{G}(y)$ $\subseteq H_{1}$. Then $d_{G}\left(x_{0}\right)+d_{G}(y) \leq 2\left(\left\lfloor\frac{|V(H)|}{2}\right\rfloor-1\right)+n-(|V(H)|+k-3) \leq n-k+1$. However, $\bar{\sigma}_{k}(G) \leq d_{G}\left(P_{0}\right) \leq d_{G}(P) \leq n-k+1+2(k-2) \leq n+k-3<\bar{\sigma}_{k}(G)$, a contradiction.

By Claim 3, $\left|V\left(H_{1}\right)\right| \leq\left\lfloor\frac{n-k+2}{2}\right\rfloor$. We have a path $P_{0}=y x_{0} \cdots x_{k-2}$, where $y \in V\left(H_{1}\right)$. Note that $d_{G}(y) \leq\left|V\left(H_{1}\right)\right|-1 \leq\left\lfloor\frac{n-k+2}{2}\right\rfloor-1$ and $d_{G}\left(x_{0}\right) \leq\left|V\left(H_{1}\right)\right|-1+1 \leq\left\lfloor\frac{n-k+2}{2}\right\rfloor$. However, $\bar{\sigma}_{k}(G) \leq d_{G}\left(P_{0}\right) \leq\left\lfloor\frac{n-k+2}{2}\right\rfloor+\left\lfloor\frac{n-k+2}{2}\right\rfloor-1+2(k-2) \leq n+k-3<\bar{\sigma}_{k}(G)$, a contradiction.

Proof of Theorem 5. Let $k \geq 3$ be an integer. For the convenience of proof, we define $k$-tribe. If $H_{0}$ is a maximal subgraph of $G$ without any branch of length more than $k-2$ such that $d_{G}\left(H_{1}, H_{0}-H_{1}\right) \leq k-2$ for every subgraph $H_{1}$ of $H_{0}$, then we call $H_{0}$ a $k$-tribe. Furthermore, we use $f_{k}(\tilde{H})$ to denote the number of $k$-tribes of a subgraph $\tilde{H}$ of $G$.

Choose a subgraph $H$ of $G$ satisfying that:

(1) $d_{H}(x) \equiv 0(\bmod 2)$ for every $x \in V(H)$;

(2) $V_{0}(H) \subseteq V_{\geq 3}(G) \subseteq V(H)$;

(3) subject to (1), (2), $|(G ; H)|$ is minimized, where $(G ; H)=\left\{H_{1} \subseteq H\right.$ : $\left.d_{G}\left(H_{1}, H-H_{1}\right) \geq k-1\right\}$

(4) subject to (1), (2), (3), $|V(H)|$ is maximized.

By Theorem 6, for $s=k-1$, it suffices to prove that $H \in E U_{k-1}(G)$. By the choice of $H, H$ satisfies Conditions (I), (II) in Theorem 6 . Since $G$ is 2-connected, $H$ satisfies Condition (V). Besides, $H$ satisfies Condition (IV). Suppose otherwise. Then $G$ has a branch $B \in \mathcal{B}(G)$ such that $|E(B)| \geq k+1$ and $E(B) \cap E(H)=\emptyset$. Then there is a path $P \subseteq B$ of order $k$ with $V(P) \subseteq V_{2}(G)$. However, since $n>6 k+3, \bar{\sigma}_{k}(G) \leq d_{G}(P)=2 k<\frac{2 n}{5}-\frac{2 k}{5}-\frac{1}{5}<\bar{\sigma}_{k}(G)$, a contradiction. Next, we prove that $H$ satisfies Condition (III), that is, $(G ; H)=\emptyset$.

We then assume that $(G ; H) \neq \emptyset$, i.e., there is a subgraph $H_{1}$ of $H$ such that $d_{G}\left(H_{1}, H-\right.$ $\left.H_{1}\right) \geq k-1$. Since $G$ is 2 -connected, there are at least two paths $B_{1}\left(x_{1}, y_{1}\right), B_{2}\left(x_{2}, y_{2}\right)$ between $H_{1}$ and $H-H_{1}$ such that $x_{1}, x_{2} \in V\left(H_{1}\right), y_{1}, y_{2} \in V\left(H-H_{1}\right)$ and $x_{1} \neq x_{2}, y_{1} \neq$ $y_{2}$. Since $d_{G}\left(H_{1}, H-H_{1}\right) \geq k-1, E\left(B_{i}\right) \bigcap E(H)=\emptyset$ and $\left|E\left(B_{i}\right)\right| \geq k-1, i=1,2$. By the choice of $H$, both $H_{1}$ and $H-H_{1}$ are the union of connected even subgraphs of $G$ and $B_{1}\left(x_{1}, y_{1}\right), B_{2}\left(x_{2}, y_{2}\right) \in \mathcal{B}(G)$.

Since $G$ is 2-connected and $B_{1}, B_{2} \in \mathcal{B}(G)$, there is a cycle $C$ with minimum order containing $B_{1}$ and $B_{2}$. Furthermore, we claim that $E(C) \cap E\left(H_{1}\right) \neq \emptyset$ or $E(C) \bigcap E\left(H-H_{1}\right) \neq \emptyset$. Otherwise, we have a subgraph $H^{1}=H \triangle C$ of $G$ satisfying (1), (2) of (3.2) and $\left|\left(G: H^{1}\right)\right|<$ $|(G: H)|$, contradicting the choice of $H$ in terms of (3) of (3.2).

Let $\tilde{H}=H-\{B \in \mathcal{B}(G):|E(B)| \geq k-1\}$. $\tilde{H}$ is the union of some $k$-tribes. In the following text, we investigate $f_{k}(\tilde{H})$. Since $(G ; H) \neq \emptyset, f_{k}(\tilde{H}) \geq 2$.

Claim 4. Either $\left\{x_{1}, x_{2}\right\}$ or $\left\{y_{1}, y_{2}\right\}$ is in distinct $k$-tribes.

Proof. Suppose otherwise. Then $H^{1}=(H \triangle C) \cup V_{\geq_{3}}(G)$ is a subgraph satisfying (1), (2) of (3.2) and $\left|\left(G ; H^{1}\right)\right|<|(G ; H)|$, contradicting the choice of $H$ in terms of (3) of (3.2).

By Claim 4 , we know $f_{k}(\tilde{H})>2$. By symmetry, we always assume that $H-H_{1}$ contains more $k$-tribes than $H_{1}$ and $\left\{y_{1}, y_{2}\right\}$ is in distinct $k$-tribes in the following text. 
Claim 5. $f_{k}(\tilde{H}) \geq 5$

Proof. Suppose otherwise. Then we consider the following three cases.

Case 1. $f_{k}(\tilde{H})=3$.

We assume that $H_{1}$ contains one $k$-tribe $\tilde{H}_{1}$ and $H-H_{1}$ contains two $k$-tribes $\tilde{H}_{2}$ and $\tilde{H}_{3}$. Then $x_{1}, x_{2} \in \tilde{H}_{1}$. By Claim 4, we assume that $y_{1} \in \tilde{H}_{2}$ and $y_{2} \in \tilde{H}_{3}$ in Case 1 .

Subcase 1.1. $H-H_{1}$ does not contain any branch connecting $\tilde{H}_{2}$ and $\tilde{H}_{3}$. By Symmetry, $|(G ; H)|=2\left(\begin{array}{l}3 \\ 1\end{array}\right)=6$. Then $H^{1}=(H \triangle C) \cup V_{\geq 3}(G)$ is a subgraph satisfying (1), (2) of (3.2) and $\left|\left(G ; H^{1}\right)\right|<|(G ; H)|$, a contradiction.

Subcase 1.2. $H-H_{1}$ contains a branch $B_{3}$ connecting $\tilde{H}_{2}$ and $\tilde{H}_{3}$.

Since $H-H_{1}$ is an even subgraph, $H-H_{1}$ contains another branch $B_{4}$ connecting $\tilde{H}_{2}$ and $\tilde{H}_{3}$. Then $(G ; H)=\left\{H_{1}, H-H_{1}\right\}$. Then $H^{1}=(H \triangle C) \cup V_{\geq 3}(G)$ is a subgraph satisfying (1), (2) of (3.2). Since $H^{1}$ contains $B_{3}$ or $B_{4},\left|\left(G ; H^{1}\right)\right|<|(G ; H)|$, a contradiction.

Case 2. $H_{1}$ contains one $k$-tribe $\tilde{H}_{1}$ and $H-H_{1}$ contains three $k$-tribes $\tilde{H}_{2}, \tilde{H}_{3}$ and $\tilde{H}_{4}$.

We assume that $y_{1} \in \tilde{H}_{2}$ and $y_{2} \in \tilde{H}_{4}$. Then we claim that $G$ has no branch between $\tilde{H}_{2}$ and $\tilde{H}_{4}$. Otherwise, $G$ contains a branch $B_{3}$ connecting $\tilde{H}_{2}$ and $\tilde{H}_{4}$. Since $G$ is 2-connected, there is a path $P\left(y_{1}, y_{2}\right)$ with minimum order such that $B_{3} \subseteq P$ and $E(P) \cap E\left(B_{1} \bigcup B_{2} \cup H_{1}\right)=\emptyset$. Note that cycle $C$ contain a path $C\left(y_{1}, y_{2}\right)$ such that $E\left(C\left(y_{1}, y_{2}\right)\right) \cap E\left(B_{1} \bigcup B_{2} \bigcup H_{1}\right)=\emptyset$. By replacing $C\left(y_{1}, y_{2}\right)$ with $P\left(y_{1}, y_{2}\right)$, we have a cycle $C_{1}$ containing $B_{1}, B_{2}$ and $B_{3}$. Then $H^{2}=\left(H \triangle C_{1}\right) \cup V_{\geq 3}(G)$ is a subgraph satisfying (1), (2) of (3.2) and $\left|\left(G ; H^{2}\right)\right|<|(G ; H)|$, a contradiction. Therefore, any $\left(y_{1}, y_{2}\right)$-path containing common vertex with $H-H_{1}$ has to contain common vertex with $\tilde{H}_{3}$. Then we distinguish the following two subcases.

Subcase 2.1. $H-H_{1}$ does not contain any branch between $\tilde{H}_{2}$ and $\tilde{H}_{3}$ or any branch between $\tilde{H}_{3}$ and $\tilde{H}_{4}$.

Then $H^{1}=(H \triangle C) \cup V_{\geq 3}(G)$ is a subgraph satisfying (1), (2) of $(3.2)$ and $\left|\left(G ; H^{1}\right)\right|<$ $|(G ; H)|$, a contradiction.

Subcase 2.2. $H-H_{1}$ contains both some branch between $\tilde{H}_{2}$ and $\tilde{H}_{3}$ and some branch between $\tilde{H}_{3}$ and $\tilde{H}_{4}$.

Since $H-H_{1}$ is an even subgraph, $H-H_{1}$ contains at least two branches between $\tilde{H}_{2}$ and $\tilde{H}_{3}$ and two branches between $\tilde{H}_{3}$ and $\tilde{H}_{4}$. Then $(G ; H)=\left\{H_{1}, H-H_{1}\right\}$. Then $H^{1}=$ $(H \triangle C) \cup V_{\geq 3}(G)$ is a subgraph satisfying (1), (2) of (3.2). Since $H^{1}$ contains at least one branch connecting $\tilde{H}_{2}$ and $\tilde{H}_{3}$ and one branch connecting $\tilde{H}_{3}$ and $\tilde{H}_{4},\left|\left(G ; H^{1}\right)\right|<|(G ; H)|$, a contradiction.

Case 3. Both $H_{1}$ and $H-H_{1}$ contain exactly two $k$-tribes, say $\tilde{H}_{1}$ and $\tilde{H}_{2}, \tilde{H}_{3}$ and $\tilde{H}_{4}$, respectively.

We claim that $x_{1}$ and $x_{2}$ lie in distinct $k$-tribes. Otherwise, $H^{1}=(H \triangle C) \cup V_{\geq 3}(G)$ is a subgraph satisfying (1), (2) of (3.2) and $\left|\left(G ; H^{1}\right)\right|<|(G ; H)|$, a contradiction. Then we assume that $x_{1} \in \tilde{H}_{1}, x_{2} \in \tilde{H}_{2}, y_{1} \in \tilde{H}_{3}$ and $y_{2} \in \tilde{H}_{4}$, respectively.

Subcase 3.1. $H$ contains a branch connecting $\tilde{H}_{1}$ and $\tilde{H}_{2}$ or connecting $\tilde{H}_{3}$ and $\tilde{H}_{4}$.

Since $H$ is an even subgraph, $H-H_{1}$ contains at least two such branches. But cycle $C$ contains at most one of these branches. Then $H^{1}=(H \triangle C) \cup V_{\geq 3}(G)$ is a subgraph satisfying (1), (2) of (3.2) and $\left|\left(G ; H^{1}\right)\right|<|(G ; H)|$, a contradiction.

Subcase 3.2. $H$ does not contain any branch between $\tilde{H}_{1}$ and $\tilde{H}_{2}$ or between $\tilde{H}_{3}$ and $\tilde{H}_{4}$. 
By symmetry, $\left.|(G ; H)|=2\left(\begin{array}{l}4 \\ 1\end{array}\right)+\left(\begin{array}{l}4 \\ 2\end{array}\right)\right)=20$. Then $H^{1}=(H \triangle C) \cup V_{\geq 3}(G)$ is a subgraph satisfying (1), (2) of $(3.2)$ and $\left|\left(G ; H^{1}\right)\right|<|(G ; H)|$, a contradiction.

Claim 6. Either each $\left(x_{1}, x_{2}\right)$-path in $H_{1}$ or each $\left(y_{1}, y_{2}\right)$-path in $H-H_{1}$ contains two branches with length at least $k-1$ such that these two branches have end vertices in a same $k$-tribe which has not any end vertex of other branches connecting other $k$-tribes in $H$.

Proof. Firstly, we prove that either each $\left(x_{1}, x_{2}\right)$-path in $H_{1}$ or each $\left(y_{1}, y_{2}\right)$-path in $H-H_{1}$ contains at least two branches with length at least $k-1$. Suppose otherwise. Let $P\left(x_{1}, x_{2}\right) \subseteq H_{1}$, $Q\left(y_{1}, y_{2}\right) \subseteq H-H_{1}$ be two paths and both $P$ and $Q$ contain exactly one branch with length at least $k-1$, say $B_{3}$ and $B_{4}$, respectively. Since $G$ is 2-connected, there exists a cycle $C_{1}$ containing $B_{3}$ and $B_{4}$ with minimum order. Note that if $B_{3} \subseteq H$ or $B_{4} \subseteq H$, since $H$ is an even subgraph, $H$ must contain another branch connecting the same two $k$-tribes. Then $H^{2}=\left(H \triangle C_{1}\right) \cup V_{\geq 3}(G)$ is a subgraph satisfying (1), (2) of (3.2) and $\left|\left(G ; H^{2}\right)\right|<|(G ; H)|$, a contradiction. Hence, either each $\left(x_{1}, x_{2}\right)$-path in $H_{1}$ or each $\left(y_{1}, y_{2}\right)$-path in $H-H_{1}$ contains at least two branches with length at least $k-1$. Without loss of generality, we assume that each $\left(y_{1}, y_{2}\right)$-path in $H-H_{1}$ contains at least two branches with length at least $k-1$. Let $P\left(y_{1}, y_{2}\right)$ be a path in $H-H_{1}$ containing two distinct branches with length at least $k-1$ $B_{3}\left(y_{3}, y_{4}\right)$ and $B_{4}\left(y_{5}, y_{6}\right)$. Let $y_{4}$ and $y_{5}$ lie in the same $k$-tribe $\tilde{H}_{0}$. We prove that there is not any branch connecting $\tilde{H}_{0}$ and other $k$-tribes in $H$ except $B_{3}$ and $B_{4}$. Suppose otherwise. Then $H^{1}=(H \triangle C) \cup V_{\geq 3}(G)$ is a subgraph satisfying (1), (2) of (3.2). Since cycle $C$ contains at most two of these branches connecting $\tilde{H}_{0}$ and other $k$-tribes in $H, H^{1}$ contains at least one. Then $\left|\left(G ; H^{1}\right)\right|<|(G ; H)|$, a contradiction.

In the following text, we always assume that each $\left(y_{1}, y_{2}\right)$-path in $H-H_{1}$ contains two branches with length at least $k-1$ such that these two branches have end vertices in a same $k$-tribe which has not any end vertex of other branches connecting other $k$-tribes in $H-H_{1}$.

Claim 7. For any vertex $y_{0} \in V(G)-\left(V(H) \bigcup V\left(B_{0}\right)\right)$, if $N_{G}\left(y_{0}\right) \subseteq V(H)$, then $N_{G}\left(y_{0}\right)$ is an independent set.

Proof. Suppose otherwise. Let $N_{G}\left(y_{0}\right)=\left\{v_{1}, v_{2}\right\}$ and $C^{1}=v_{1} v_{2} y_{0} v_{1}$. We have a subgraph $H^{1}=H \triangle C^{1}$ of $G$ satisfying (1), (2), (3) of (3.2) and $\left|H^{1}\right|>|H|$, contradicting the choice of $H$ in terms of (4) of (3.2).

In the following text, we will consider two cases, $f_{k}(\tilde{H})=5$ and $f_{k}(\tilde{H})>5$, respectively.

Firstly, we consider the case when $f_{k}(\tilde{H})=5$. We first consider the case when $H_{1}$ contains two $k$-tribes $\tilde{H}_{1}$ and $\tilde{H}_{2}$, and that $H-H_{1}$ contains three $k$-tribes $\tilde{H}_{3}, \tilde{H}_{4}$ and $\tilde{H}_{5}$. By Claims 4 and 5, we assume that $x_{1} \in \tilde{H}_{1}, x_{2} \in \tilde{H}_{2}, y_{1} \in \tilde{H}_{3}$ and $y_{2} \in \tilde{H}_{4}$. Then $H^{1}=(H \triangle C) \cup V_{\geq 3}(G)$ is a subgraph satisfying (1),(2) of $(3.2)$ and $\left|\left(G ; H^{1}\right)\right|<|(G ; H)|$, a contradiction.

Therefore, it remains to consider the case when $H_{1}$ contains one $k$-tribe $\tilde{H}_{1}$ and $H-H_{1}$ contains four $k$-tribes $\tilde{H}_{2}, \tilde{H}_{3}, \tilde{H}_{4}$ and $\tilde{H}_{5}$. We assume that $y_{1} \in \tilde{H}_{2}$ and $y_{2} \in \tilde{H}_{4}$. By Claim 6 , $H$ contains four branches, say $B_{3}, B_{4}, B_{5}, B_{6}$, connecting $\tilde{H}_{2}$ and $\tilde{H}_{3}, \tilde{H}_{2}$ and $\tilde{H}_{5}, \tilde{H}_{4}$ and $\tilde{H}_{3}$, $\tilde{H}_{2}$ and $\tilde{H}_{5}$, respectively. We claim that $G$ does not contain any other branch connecting two different $k$-tribes. Suppose otherwise. Firstly, we consider the case when $G$ contains another branch $B_{0}$ between $H_{1}$ and $H-H_{1}$. Since $G$ is 2-connected, there exist two cycles $C_{1}$ and $C_{2}$ such that $B_{0}, B_{1} \subseteq C_{1}$ and $B_{0}, B_{2} \subseteq C_{2}$. Without loss of generality, we assume that $\left|C_{1}\right| \geq\left|C_{2}\right|$. Then $H^{2}=\left(H \triangle C_{1}\right) \cup V_{\geq 3}(G)$ is a subgraph satisfying (1), (2) of (3.2) and $\left|\left(G ; H^{2}\right)\right|<|(G ; H)|$, a contradiction. By symmetry, it remains to consider the case when $G$ contains another branch 
connecting two different $k$-tribes of $H-H_{1}$. Then $H^{1}=(H \triangle C) \cup V_{\geq 3}(G)$ is a subgraph satisfying (1), (2) of (3.2) and $\left|\left(G ; H^{1}\right)\right|<|(G ; H)|$, a contradiction.

Without loss of generality, we assume that $\tilde{H}_{2}$ has the minimum order among the five $k$ tribes. Let $B_{3}=y_{0} y_{1} \cdots y_{l}$, where end vertex $y_{0} \in H_{2}$ and $l \geq k-1$. Since $G$ is 2-connected, $y_{0}$ belongs to at most two branches with length at least 2 . Then $d_{G}\left(y_{0}\right) \leq\left|V\left(\tilde{H}_{2}\right)\right|-1+2 \leq$ $\frac{n-6(k-2)}{5}-1+2$. We claim that $N_{G}\left(y_{0}\right) \cap V_{2}(G)=\left\{y_{1}\right\}$. Suppose otherwise. Then there exists a vertex $y \in N_{G}\left(y_{0}\right)-\left\{y_{1}\right\}$ with $d_{G}(y)=2$, and that $P=y y_{0} \cdots y_{k-2}$ a path of order $k$. Since $n>6 k+3, \bar{\sigma}_{k}(G) \leq d_{G}(P) \leq \frac{n-6(k-2)}{5}+1+2(k-1)<\frac{2 n}{5}-\frac{2 k}{5}+\frac{4}{5} \leq \bar{\sigma}_{k}(G)$, a contradiction. Then $d_{G}\left(y_{0}\right) \leq \frac{n-6(k-2)}{5}$. We consider the path $Q=x y_{0} \cdots y_{k-2}$, where $x \in V\left(\tilde{H}_{2}\right)$. By Claim 7, $d_{G}(x) \leq\left|V\left(\tilde{H}_{2}\right)\right|-1 \leq \frac{n-6(k-2)}{5}-1$. Then $\bar{\sigma}_{k}(G) \leq d_{G}(P) \leq$ $\frac{n-6(k-2)}{5}+\frac{n-6(k-2)}{5}-1+2(k-2)=\frac{2 n}{5}-\frac{2 k}{5}-\frac{1}{5}<\bar{\sigma}_{k}(G)$, a contradiction.

Therefore, $f_{k}(\tilde{H}) \neq 5$. Next, we consider the case when $f_{k}(\tilde{H})>5$.

By Claim 4 , we assume that $y_{1} \subseteq \tilde{H}_{2}$ and $y_{2} \subseteq \tilde{H}_{4}$. Let $H_{2} \supseteq \tilde{H}_{2}$ be a maximal subgraph of $H$ satisfying the condition that if $H_{2}$ contains a $k$-tribe $\tilde{H}^{0} \neq \tilde{H}_{2}$, then $H_{2}$ contains another $k$-tribe $\tilde{H}^{1}$ such that there are at least two branches between $\tilde{H}^{0}$ and $\tilde{H}^{1}$ in $G$. And let $H_{4} \supseteq \tilde{H}_{4}$ be a maximal subgraph of $H$ satisfying the condition that if $H_{4}$ contains a $k$-tribe $\tilde{H}^{0} \neq \tilde{H}_{2}$, then $H_{4}$ contains another $k$-tribe $\tilde{H}^{1}$ such that there are at least two branches between $\tilde{H}^{0}$ and $\tilde{H}^{1}$ in $G$. We claim that $V\left(H_{2}\right) \cap V\left(H_{4}\right)=\emptyset$. Suppose otherwise. Then $H_{2}=H_{4}$. Then $H^{1}=(H \triangle C) \cup V_{\geq 3}(G)$ is a subgraph satisfying (1), (2) of $(3.2)$ and $\left|\left(G ; H^{1}\right)\right|<|(G ; H)|$, a contradiction. Since $G$ is 2-connected, there are at least two paths connecting $H_{2}$ and $H_{4}$.

Claim 8. Each path connecting $H_{2}$ and $H_{4}$ contains two branches with length at least $k-1$ such that these two branches have end vertices in a same subgraph which has not any end vertex of other branches connecting other $k$-tribes in $G$.

Proof. We first prove that each path connecting $H_{2}$ and $H_{4}$ contains at least two branches with length at least $k-1$. Suppose otherwise. Then $H^{1}=(H \triangle C) \cup V_{\geq 3}(G)$ is a subgraph satisfying (1), (2) of $(3.2)$ and $\left|\left(G ; H^{1}\right)\right|<|(G ; H)|$, a contradiction.

Hence, let $P$ be a path in $H-H_{1}$ containing two distinct branches $B_{3}\left(y_{3}, y_{4}\right), B_{4}\left(y_{5}, y_{6}\right)$ and $\left\{y_{4}, y_{5}\right\}$ lies in the same subgraph $H_{3}$. We prove that there is not any branch connecting $H_{2}$ and $H_{3}$ or $H_{3}$ and $H_{4}$ in $G$ except $B_{3}$ or $B_{4}$. Suppose otherwise. Then $H^{1}=(H \triangle C) \cup V_{\geq 3}(G)$ is a subgraph satisfying (1), (2) of $(3.2)$ and $\left|\left(G ; H^{1}\right)\right|<|(G ; H)|$, a contradiction.

By Claim 8, we assume that $H_{3}$ is a subgraph of $H-H_{1}$, and that $B_{3}$ and $B_{4}$ are two branches connecting $H_{2}$ and $H_{3}, H_{3}$ and $H_{4}$, respectively. We claim that $B_{3}, B_{4} \subseteq H-H_{1}$. Suppose otherwise. Then $H^{1}=(H \triangle C) \cup V_{\geq 3}(G)$ is a subgraph satisfying (1), (2) of (3.2) and $\left|\left(G ; H^{1}\right)\right|<|(G ; H)|$, a contradiction. Since $H-H_{1}$ is an even subgraph, $H-H_{1}$ contains a subgraph $H_{5}$ and two branches $B_{5}$ and $B_{6}$ such that $B_{5}$ connects $H_{2}$ and $H_{5}$, and $B_{6}$ connects $H_{4}$ and $H_{5}$, respectively. We claim $V\left(H_{3}\right) \cap V\left(H_{5}\right)=\emptyset$. Otherwise, $H_{3}=H_{5}$ is the same subgraph and then $H^{1}=(H \triangle C) \cup V_{\geq 3}(G)$ is a subgraph satisfying (1), (2) of (3.2), hence $\left|\left(G ; H^{1}\right)\right|<|(G ; H)|$, a contradiction. Furthermore, we claim that $G$ does not contain any other branch between subgraphs $H_{1}, H_{2}, H_{3}, H_{4}$ and $H_{5}$. Suppose otherwise. Firstly, we consider the case when $G$ contains another branch $B_{0}$ between $H_{1}$ and $H-H_{1}$. Since $G$ is 2-connected, there exist two cycles $C_{1}$ and $C_{2}$ such that $B_{0}, B_{1} \subseteq C_{1}$ and $B_{0}, B_{2} \subseteq C_{2}$. By symmetry, we assume that $\left|C_{1}\right| \geq\left|C_{2}\right|$. Then $H^{2}=\left(H \triangle C_{1}\right) \cup V_{\geq 3}(G)$ is a subgraph satisfying (1), (2) of (3.2) and $\left|\left(G ; H^{2}\right)\right|<|(G ; H)|$, a contradiction. It remains to consider the case when $G$ contains 
another branch connecting two different subgraphs of $H-H_{1}$. Then $H^{1}=(H \triangle C) \cup V_{\geq 3}(G)$ is a subgraph satisfying $(1),(2)$ of $(3.2)$ and $\left|\left(G ; H^{1}\right)\right|<|(G ; H)|$, a contradiction.

Without loss of generality, we assume that $H_{2}$ has the minimum order among the five subgraphs. Let $B_{3}=y_{0} y_{1} \cdots y_{l}$, where end vertex $y_{0} \in H_{2}$ and $l \geq k-1$. Since $G$ is 2 -connected, $y_{0}$ belongs to at most two branches with length at least 2 . Then $d_{G}\left(y_{0}\right) \leq\left|V\left(H_{2}\right)\right|-1+2 \leq$ $\frac{n-6(k-2)}{5}-1+2$. Furthermore, we claim that $N_{G}\left(y_{0}\right) \bigcap V_{2}(G)=\left\{y_{1}\right\}$. suppose otherwise. Then there exists a vertex $y \in N_{G}\left(y_{0}\right)-\left\{y_{1}\right\}$ with $d_{G}(y)=2$, and that $P=y y_{0} \cdots y_{k-2}$ is a path of order $k$. Since $n>6 k+3, \bar{\sigma}_{k}(G) \leq d_{G}(P) \leq \frac{n-6(k-2)}{5}+1+2(k-1)<\frac{2 n}{5}-\frac{2 k}{5}+\frac{4}{5} \leq \bar{\sigma}_{k}(G)$, a contradiction. Then $d_{G}\left(y_{0}\right) \leq \frac{n-6(k-2)}{5}$. We consider the path $Q=x y_{0} \cdots y_{k-2}$, where $x \in V\left(H_{2}\right)$. By Claim $7, d_{G}(x) \leq\left|V\left(H_{2}\right)\right|-1 \leq \frac{n-6(k-2)}{5}-1$. Then $\bar{\sigma}_{k}(G) \leq d_{G}(P) \leq$ $\frac{n-6(k-2)}{5}+\frac{n-6(k-2)}{5}-1+2(k-2)=\frac{2 n}{5}-\frac{2 k}{5}-\frac{1}{5}<\bar{\sigma}_{k}(G)$, a contradiction.

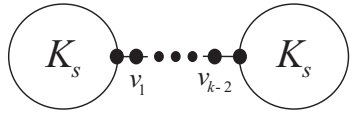

$G_{1}$

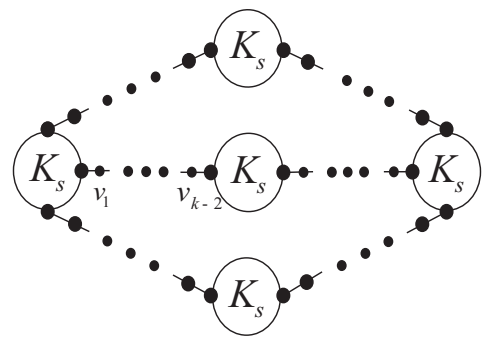

$G_{2}$

Figure 1. $G_{1}$ and $G_{2}$.

\section{$\S 4$ Conclusion: Sharpness}

Both Theorems 4 and 5 are best possible, this may be seen by $G_{1}$ and $G_{2}$ in Figure 1 , respectively.

Comparing Theorem 2 with Theorem 4, one might think that they would have a unified bound. Unfortunately, this is not true: Theorem 4 is not direct promotion of Theorem 2 . However, taking the Dirac result and Theorems 2, 3, 4 and 5 into consideration, we conclude that we completely know the sharp lower bounds of $\bar{\sigma}_{k}(G)$ involving its order for the graph $L^{k-1}(G)$ of a (2-)connected graph $G$ to be hamiltonian for all $k \geq 1$.

\section{Acknowledgement}

We would like to express our sincere appreciation to reviewers and editors and thanks for their careful reading and valuable revision recommendations.

Open Access This article is licensed under a Creative Commons Attribution 4.0 International License, which permits use, sharing, adaptation, distribution and reproduction in any medium or format, as long as you give appropriate credit to the original author(s) and the source, provide a link to the Creative Commons licence, and indicate if changes were made. The 
images or other third party material in this article are included in the articles Creative Commons licence, unless indicated otherwise in a credit line to the material. If material is not included in the articles Creative Commons licence and your intended use is not permitted by statutory regulation or exceeds the permitted use, you will need to obtain permission directly from the copyright holder. To view a copy of this licence, visit http://creativecommons.org/licenses/by/4.0/.

\section{References}

[1] A Benhocine, L Clark, N Köhler, H J Veldman. On circuits and pancyclic line graphs, J Graph Theory, 1986, 10(3): 411-425.

[2] J A Bondy, U S R Murty. Graph Theory, New York: Springer, 2008.

[3] R A Brualdi, R F Shanny. Hamiltonian line graphs, J Graph Theory, 1981, 5: 307-314.

[4] P A Catlin, I T N Janakiraman, N Srinivasan. Hamilton cycles and closed trails in iterated line graphs, J Graph Theory, 1990, 14: 347-364.

[5] G Chartrand. On hamiltonian line graphs, Trans Amer Math Soc, 1968, 134(4): 559-566.

[6] L Clark. On hamiltonian line graphs, J Graph Theory, 1984, 8(2): 303-307.

[7] G A Dirac. Some theorems on abstract graphs, Proc London Math Soc, 1952, 2: 69-81.

[8] Z Ryjáček, G J Woeginger, L M Xiong. Hamiltonian index is NP-complete, Discrete Math, 2011, 159(4): 246-250.

[9] M L Saražin. A simple upper bound for the hamiltonian index of a graph, Discrete Math, 1994, 134(1-3): 85-91.

[10] H J Veldman. On dominating and spanning circuits in graphs, Discrete Math, 1994, 124(1-3): 229-239.

[11] L M Xiong. The Hamiltonian index of a graph, Graphs Combin, 2001, 17(4): 775-784.

[12] L M Xiong, H J Broersma, X L Li, M C Li. The Hamiltonian index of a graph and its branchbonds, Discrete Math, 2004, 285(1-3): 279-288.

[13] L M Xiong, Z H Liu. Hamiltonian iterated line graphs, Discrete Math, 2002, 256(1-2): 407-422.

[14] L M Xiong, Z Ryjacek, H J Broersma. On stability of the hamiltonian index under contractions and closures, J Graph Theory, 2005, 49: 104-115.

[15] L M Xiong, Q Q Zhu. The hamiltonian index of a graph-A survey, J Jiangxi Normal University (Natural Science), 2014, 38: 229-235.(in Chinese)

${ }^{1}$ School of Mathematics and Statistics, Beijing Institute of Technology, Beijing 100081, China.

Email: zemengliu@foxmail.com

${ }^{2}$ School of Mathematics and Statistics, Beijing Key Laboratory on MCAACI, Beijing Institute of Technology, Beijing 100081, China.

Email: lmxiong@bit.edu.cn 